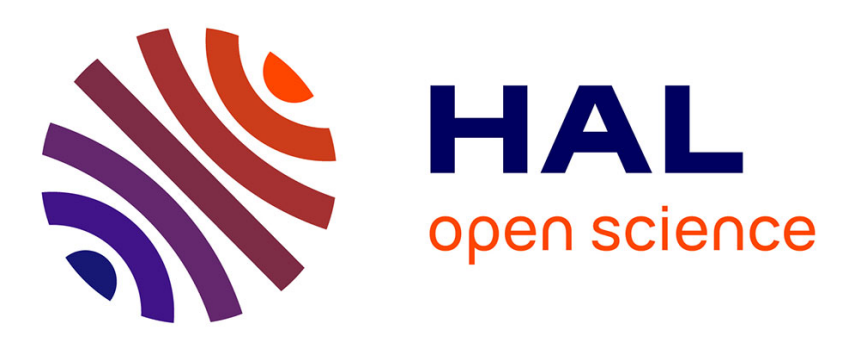

\title{
Image-Based Lateral Position, Steering Behavior Estimation, and Road Curvature Prediction for Motorcycles
}

\author{
Pierre-Marie Damon, Hicham Hadj-Abdelkader, Hichem Arioui, Kamal \\ Youcef-Toumi
}

\section{To cite this version:}

Pierre-Marie Damon, Hicham Hadj-Abdelkader, Hichem Arioui, Kamal Youcef-Toumi. Image-Based Lateral Position, Steering Behavior Estimation, and Road Curvature Prediction for Motorcycles. IEEE Robotics and Automation Letters, 2018, 3 (3), pp.2694-2701. 10.1109/LRA.2018.2831260 . hal01933660

\section{HAL Id: hal-01933660 https://hal.science/hal-01933660}

Submitted on 4 Oct 2019

HAL is a multi-disciplinary open access archive for the deposit and dissemination of scientific research documents, whether they are published or not. The documents may come from teaching and research institutions in France or abroad, or from public or private research centers.
L'archive ouverte pluridisciplinaire HAL, est destinée au dépôt et à la diffusion de documents scientifiques de niveau recherche, publiés ou non, émanant des établissements d'enseignement et de recherche français ou étrangers, des laboratoires publics ou privés. 


\title{
Image-Based Lateral Position, Steering Behavior Estimation and Road Curvature Prediction for Motorcycles
}

\author{
Pierre-Marie Damon ${ }^{1,2}$, Hicham Hadj-Abdelkader ${ }^{1}$, Hichem Arioui ${ }^{1}$, and Kamal Youcef-Toumi ${ }^{2}$
}

\begin{abstract}
This paper presents an image-based approach to simultaneously estimate the lateral position of a powered-two wheeled vehicle on the road, its steering behavior and predict the road curvature ahead of the motorcycle. This work is based on the inverse perspective mapping technique combined with a road lanes detection algorithm capable of detecting straight and curved lanes. Then, a clothoid model is used to extract pertinent information from the detected road markers. Finally, the performance of the proposed approach is illustrated through simulations carried out with the well-known motorcycle simulator "BikeSim". The results are very promising since the algorithm is capable of estimating, in real time, the road geometry and the vehicle location with a better accuracy than the one given by the commercial GPS.
\end{abstract}

\section{INTRODUCTION}

Nowadays, the transport of people and merchandise is a major concern in our societies. The transportation usage is constantly changing with new technologies. Even if new generation of vehicles is emerging on the market like autonomous cars, Powered Two-Wheeled Vehicles (P2WV) still stay as the most popular around the world. In highincome countries, motorcycles are considered more as luxury goods whereas in low and middle-income regions they are an essential means of transportation. This is due to their affordable price and their low fuel consumption. In addition, their handling capabilities and their compact design allow riders to easily escape the traffic jams and to ride on any kind of road where the use of Powered Four-Wheeled Vehicles (P4WV) is sometimes impossible.

Although, the use of P2WV is different from one country to another, the road accidents involving motorcycle users is a global matter. For example, during the year 2016, the U.S. Department of Transportation National Highway Traffic Safety Administration (NHTSA) reported 37461 people killed in crashes on U.S. roads including 5286 motorcyclists [1]. According to these statistics the global number of road accidents and related fatalities is quasi-stable for several years except for P2WV where the number of motorcyclist fatality counts is the highest since 2008. Paradoxically, P2WV represented less than $1 \%$ of the total travel distance including all kind of vehicles, around 3\% of the registered vehicles against $14 \%$ of all road deaths and around of 90000

1 Pierre-Marie Damon, Hicham Hadj-Abdelkader and Hichem Arioui are with IBISC Laboratory, University of Paris-Saclay, France. \{pierremarie.damon, hicham.hadjabdelkader, hichem.arioui\}euniv-evry.fr

2 Pierre-Marie Damon and Kamal Youcef-Toumi are with Mechatronics Research Laboratory (MRL), Massachusetts Institute of Technology, USA \{pdamon, youcef\}amit.edu

This work was supported by VIROLO++ motorcyclists injured. All these figures explicitly highlight that P2WV users are the most vulnerable road users in the US. Unfortunately, this finding is the same for many areas and much worse in several countries like Malaysia where more than $50 \%$ of the road deaths are motocyclists.

In most of the vehicles crashes, investigations have shown that loss of control often happened and for most of them, they are due to inappropriate driver action. That is why during the last decades, the development of Advance Driver Assistance Systems (ADAS) was a priority in automotive industry. It has largely contributed to decreasing the number of fatal accidents while increasing comfort in our vehicles. Nevertheless, the development of Advance Rider Assistance Systems (ARAS) dedicated to P2WV did not follow the same growth even if few solutions currently exist on the market for premium motorcycles. The cost of such systems, the high complexity of motorcycle dynamics, the integration constrains due to the compact design are some of the reasons that slow down their development. Indeed, P4WV and P2WV dynamics are different because of the large roll motion and the extreme load transfer phenomena. Hence, it requires the development of specific ARAS for motorcycles.

Negotiating a turn when riding a motorcycle can be very tricky for a novice rider. When approaching a curve, he must follow a safe trajectory by adapting the speed and acting on the handlebar to properly lean the vehicle. A slight mistake often leads to a crash. Even for experienced riders the curves can be dangerous because of the road infrastructure defaults or the environment conditions (sandy road, friction changes, pothole, etc.). In [2], authors studied the effect of horizontal curvature on P2WV accidents along rural highways. This study clearly shows that the horizontal road geometry impacts the frequency and the severity of P2WV crashes. Among the most significant factors, the authors mentioned the radius of the curve and its length. In [3], a similar study has been carried out in UK. The statistics show that the curve accidents represent around $15 \%$ of all motorcycle fatalities and riders involved in this type of accident are nearly three times less experienced.

All these findings highlight that adequate ARAS could undoubtedly decrease the number of accidents in turns because they are mainly due to riding action errors. The current work aims to address this concern and propose a method to simultaneously estimate the motorcycle lateral position, its heading angle relative to the road and predict the road curvature at a look ahead distance of the vehicle. Moreover, the solution is very affordable since it only requires two basic sensors which are a simple monocular camera and an Inertial 
Measurement Unit (IMU).

This paper is organized as follows. Section 2 presents the motivations and defines the problem. The inverse perspective mapping technique and the road marker filtering is addressed in section 3. Whereas in section 4, we will discuss the algorithm performing the road curvature prediction, the P2WV lateral position and heading estimation. Then in section 5, simulation results of carried out with the motorcycle simulator BikeSim are discussed. Finally, concluding remarks are summarized in section 6 .

\section{MOTIVATION AND PROBLEM STATEMENT}

Since the recent progress in computing capabilities of vehicle electronic control unit and in the embedded network speed (CAN, Ethernet, etc.), Computer Vision (CV) algorithms have been largely used in ADAS. They deal with a wide range of applications such that the lane keeping assist systems [4], the obstacle detection [5], the parking assistance, etc. Other works have addressed the estimation of the vehicle dynamics as in [6] were the authors used vision to estimate the vehicle Euler angles. Finaly, soon, cameras could replace most of the embedded sensors such that gyroscopes, accelerometers, wheel speed sensors, etc. Although the CV research community working on P4WV is very active, only few works deal with motorcycle applications. In [7], the authors introduced a smartphone-based rider assistance system to detect obstacles ahead of a scooter. In [8], [9], [10], the authors proposed similar works inspired by the Simultaneous Localization And Mapping (SLAM) technique to reconstruct the motorcycle trajectory and estimate the camera egomotion. In [11], [12], the authors introduced a method and its validation to estimate the roll angle with a monocular camera. All these works demonstrate that camera turns out to be a very important sensor in the development of ADAS and ARAS. Indeed, the camera allows one to get simultaneous information about the vehicle location in its environment and its dynamics (speed, orientation, etc.).

This work proposes a vision-based method to contribute to the development of ARAS. Note that only the estimation part is addressed in this paper. To fully design an ARAS this work needs to be completed with risk function design and a rider alert interface for passive safety systems such as a Human-Machine Interface (HMI) or a vibrating handlebar. In case of semi-active and active system design, the passive solution must be completed with actuators and adequate control algorithms. The proposed solution is based on a simple hardware architecture regarding the objectives which are to simultaneously predict the road geometry, estimate the lateral position and the steering behavior of the P2WV on the road. It uses only two basic sensors, namely a simple monocular camera and an Inertial Measurement Unit (IMU). Note that the estimation accuracy directly depends on the camera resolution and the quality of the road markers but in general the results are much more precise than those given by a common GPS.

Estimates of the three pieces of information are crucial when approaching a curve. The driver needs to adapt the speed and the motorcycle position on the road to properly negociate the turn. In [13], a P2WV curve warning system was proposed for the first time. It continuously computes the ideal maneuver from the actual state to detect dangerous situations. Nevertheless, the method requires exact knowledge of the road map of and the accurate position of the motorcycle. These assumptions are very restrictive because even if we consider a world road map is available, equip a commercial motorcycle with an affordable and accurate GPS is very difficult. In this paper, we propose a solution which bypasses these requirements by using the inverse perspective mapping (IPM) technique. It computes a Bird-Eye-View (BEV) of a given frame. Furthermore, the perceptive effect coming from the camera projective transformation is removed, making the geometric properties of the road markers easily identibiable. This method is largely used in various kind of automotive applications. Note that all the algorithms proposed for P4WV can not be extended to P2WV because none of them takes into account the roll motion which is vital when considering motorcycles. In this context, we can cite many works dealing with P2WV roll angle estimation: [14], [15], [16], [17]. In [7], the author used the IPM approach to synthesize an obstacle detection algorithm for P2WV with a smart-phone. Nevertheless, due to limited computing power of the device, the IPM is approximated requiring a look up table and a calibration step. Consequently, this method is approximate since it depends on the look up table accuracy and how the user places the smart-phone on the motorcycle. In [18], the author proposed an observer to estimate the road curvature for P2WV. Nevertheless, the solution is only able to estimate the road curvature at the actual state and has not been tested on a simulator or on real data.

The main contribution of this work is the development of a vision-based algorithm allowing the simultaneous prediction and estimation of crucial information for ARAS design. These information are the road curvature, the vehicle lateral position on the road and the vehicle steering behavior. The latter corresponds to the relative yaw motion between the road and the vehicle trajectories and easily allows the detection of over or under-steering behavior. The algorithm is able to predict the road curvature around $30 \mathrm{~m}$ ahead of the vehicle. This means that for a passive alert system at 50 $\mathrm{km} / \mathrm{h}$ the rider has more than 2 seconds to react whereas at $100 \mathrm{~km} / \mathrm{h}$ he has around 1 second. Of course, this algorithm could be used in more advance active and semi-active safety systems too. Although an accurate map of the road combined with a high-performance GPS could give similar information as in [13], the proposed solution is much more affordable since it requires only low cost sensors. In addition, it does not require any vehicle or tire model as in [18].

\section{INVERSE PERSPECTIVE MAPPING AND LANE DETECTION}

\section{A. Inverse perspective mapping}

The Inverse Perspective Mapping (IPM) aims to create a Bird's-Eye-View (BEV) image of the road as depicted in step 1 on Figure 1. Let us consider a conventional camera 
attached to frame $\mathcal{F}_{c}$. Its intrinsic parameters are given by the calibration matrix $\mathbf{K}$ whereas its extrinsic ones are given by the rotation matrix $\mathbf{R} \in \mathbb{S O}(3)$ and the translation vector $\mathbf{t} \in \mathbb{R}^{3}$. For the sake of simplicity, the camera distortions are not considered nevertheless one can note that distortion parameters can be obtained after camera calibration. Let $P$ be a $3 \mathrm{D}$ point with homogeneous coordinates $\mathbf{P}_{w}=$ ( $\left.\begin{array}{lll}X & Y & Z\end{array}\right)^{\top}$ in the world frame $\mathcal{F}_{w}$. The image formation of the 3D point $P$ is obtained through its projection into the $2 \mathrm{D}$ point of homogeneous coordinates $\mathbf{p}=\left(\begin{array}{lll}u & v & 1\end{array}\right)^{\top}$ in the image plane $\mathcal{F}_{i}$. The projection equation is given by:

$$
\mathbf{p} \propto \mathbf{K}\left(\begin{array}{ll}
\mathbf{R} & \mathbf{t}
\end{array}\right) \mathbf{P}_{w}
$$

where $\propto$ denotes the equality up to scale.

The camera calibration matrix $\mathbf{K}$ is given by:

$$
\mathbf{K}=\left(\begin{array}{ccc}
f_{u} & 0 & u_{0} \\
0 & f_{v} & v_{0} \\
0 & 0 & 1
\end{array}\right)
$$

where $u_{0}$ and $v_{0}$ are the pixel coordinates of the principal point. $f_{u}$ and $f_{v}$ are the horizontal and vertical focal lengths which can be expressed in terms of the horizontal and vertical field of view (FOV) respectively denoted $F O V_{u}$ and $F O V_{v}$ such that $f_{u}=u_{0} \tan ^{-1}\left(F O V_{u} / 2\right)$ and $f_{v}=$ $v_{0} \tan ^{-1}\left(F O V_{v} / 2\right)$. As for distortion, these intrinsic parameters can be obtained after a calibration step.

As discussed in the previous section, the IPM is a common technique used to get a top-view image of an input frame. In other words the IPM creates a virtual camera removing the perspective effect coming from equation (1). Let us consider a point $p$ with homogeneous coordinates $\mathbf{p}=\left(\begin{array}{lll}u & v & 1\end{array}\right)^{\top}$ belonging to an input image $I$. The IPM transforms $p$ into a new point $p^{\prime}$ of homogeneous coordinates $\mathbf{p}^{\prime}=\left(u^{\prime} v^{\prime} 1\right)^{\top}$ in the BEV image $I^{\prime}$. The transformation equation is given by:

$$
\mathbf{p}^{\prime} \propto \mathbf{G p}
$$

where $\mathbf{G}$ is the collineation matrix ensuring the IPM transformation from $I$ to $I^{\prime}$. $\mathbf{G}$ can be expressed in terms of the intrinsic matrix $\mathbf{K}$ and the Euclidean homography matrix $\mathbf{H} \in \mathbb{S L}(3)$ related to a planar viewed object. Note that $\mathbf{H}$ depends on the rotation matrix $\mathbf{R}$ and the translation vector $\mathbf{t}$ corresponding to the rigid transformation between the real camera pose providing the image $I$ and the virtual one where $I^{\prime}$ is generated. The homography matrix can be computed using the following equation [19]:

$$
\mathbf{H}=\mathbf{R}+\frac{1}{d} \mathbf{t}^{\top}
$$

where $\mathbf{n}$ is the normal vector to the planar object, the road in our case, expressed in the virtual camera frame. Whereas $d$ is the distance between the plane and the virtual camera frame center. In case of the IPM transformation, $\mathbf{n}=\left(\begin{array}{lll}0 & 0 & 1\end{array}\right)^{\top}$ and $d$ is fixed with respect to the defined Region Of Interest (ROI) of the BEV image.

In the context of this work, the real camera is rigidly fixed on the P2WV main body and not affected by the steering motion. We assume that the camera pitch and roll angles are denoted respectively by $\mu$ and $\phi$. Let us consider these are measured by means of an IMU installed on the camera. Note that $\mu=\mu_{0}+\delta \mu$, with $\mu_{0}$ is the camera mounting pitch angle and $\delta \mu$ is the pitch variation. Moreover, $h_{c}=z_{c} \cos (\phi)$ is the camera height above the ground level, with $z_{c}$ is the camera mounting height. Note that $\mu_{0}$ and $z_{c}$ are obtained when $\phi=0^{\circ}$. The relative yaw angle between the vehicle and the road trajectory can not be measured since, in our case, the road map is not available. Nevertheless, they are supposed to be the very close since the rider follows the road. But in case of over or under-steering regarding the road curvature, it results in a BEV image rotation around its virtual optical axis ( $Z$-axis) which is perpendicular to the ground and does not impact the algorithm derived in the next section. Finally, the rotation matrix $\mathbf{R}$ is given by $\mathbf{R}=\mathbf{R}_{\mathbf{c}} \mathbf{R}_{\phi} \mathbf{R}_{\mu}$ with $\mathbf{R}_{\mathbf{c}}$ the fixed rotation matrix to align the camera $Z$-axis with the longitudinal $X$-axis of the vehicle coordinate system. $\mathbf{R}_{\phi}$ and $\mathbf{R}_{\mu}$ are the rotation matrices respectively associated to the roll and pitch motions. Their expressions are given by:

$$
\begin{gathered}
\mathbf{R}_{c}=\left(\begin{array}{ccc}
0 & -1 & 0 \\
0 & 0 & -1 \\
1 & 0 & 0
\end{array}\right), \mathbf{R}_{\phi}=\left(\begin{array}{ccc}
1 & 0 & 0 \\
0 & \cos (\phi) & -\sin (\phi) \\
0 & \sin (\phi) & \cos (\phi)
\end{array}\right) \\
\text { and } \mathbf{R}_{\mu}=\left(\begin{array}{ccc}
\cos (\mu) & 0 & \sin (\mu) \\
0 & 1 & 0 \\
-\sin (\mu) & 0 & \cos (\mu)
\end{array}\right)
\end{gathered}
$$

Under the assumptions given above, the IPM transformation based on the collineation matrix $\mathbf{G}$ depends only on rotation matrices $\mathbf{R}_{\phi}$ and $\mathbf{R}_{\mu}$, the camera calibration matrix $\mathbf{K}$ (depending on the selected ROI and the output resolution) and its height $h_{c}$. In what follows, to facilitate the filtering and fitting steps we will assume that the BEV image $I^{\prime}$ is of size $(m \times n)$ since the original image $I$ is of size $(n \times m)$. The ROI is defined by its top-left and the bottom-right points of coordinates $\left(X_{\max }, Y_{\max }\right)$ and $\left(X_{\min }, Y_{\min }\right)$ in the vehicle frame $\mathcal{F}_{v}$ (see Figure 1).

The coordinates of $p$ given in equation (3) can be rewritten as follows:

$$
\left\{\begin{array}{l}
u=\frac{\left(-f_{u} \mathrm{~s}_{\phi} \mathrm{s}_{\mu}+u_{0} \mathrm{c}_{\mu}\right) \mathcal{X}-f_{u} \mathrm{c}_{\phi} \mathcal{Y}+\left(f_{u} \mathrm{~s}_{\phi} \mathrm{c}_{\mu}+u_{0} \mathrm{~s}_{\mu}\right) \mathcal{Z}}{\mathrm{c}_{\mu} \mathcal{X}+\mathrm{s}_{\mu} \mathcal{Z}} \\
v=\frac{\left(-f_{v} \mathrm{c}_{\phi} \mathrm{s}_{\mu}+v_{0} \mathrm{c}_{\mu}\right) \mathcal{X}+f_{v} \mathrm{~s}_{\phi} \mathcal{Y}+\left(f_{v} \mathrm{c}_{\phi} \mathrm{c}_{\mu}+v_{0} \mathrm{~s}_{\mu}\right) \mathcal{Z}}{\mathrm{c}_{\mu} \mathcal{X}+\mathrm{s}_{\mu} \mathcal{Z}}
\end{array}\right.
$$

with $\mathcal{X}=X_{\max }-u^{\prime} \frac{X_{\max }-X_{\min }}{m}, \mathcal{Y}=Y_{\max }-v^{\prime} \frac{Y_{\max }-Y_{\min }}{n}$ and $\mathcal{Z}=h_{c}$. For the sake of simplicity, the notations $\mathrm{s}_{\diamond}$ and $\mathrm{c}_{\diamond}$ denote respectively $\sin (\diamond)$ and $\cos (\diamond)$.

In other words, the BEV image $I^{\prime}$ is obtained by warping each point in $I^{\prime}$ onto $I$ using the inverse mapping $\mathbf{G}^{-1}$ and then the image intensity is computed by interpolating the local pixels intensities in the origin image $I$. An example of $\mathrm{BEV}$ image is shown in Figure 1.

\section{B. Road markers detection}

This section addresses the road markers filtering which is divided into 2 main steps. The first one consists of the detection of raw lane markers and returns a binary image 
(steps 2 to 4 shown in Figure 1). The second step separates the road lanes (step 5 in Figure 1) using a sliding window technique.

The road marker detection has been widely addressed in the past years. In this work, we use the method initially introduced in [20] and revisited in [21]. This algorithm is based on the intensity contrast between the road lane markers and their surroundings. It allows the detection of both straight and curved road lanes and requires less computer resources than other method like those using the well-known RANSAC. Moreover, this method turns out to be a very appropriate solution to detect the road lanes on a BEV image since it takes into account the line width, denoted $w$, as a filtering parameter. Note that the road markers properties (width, length, etc.) are precisely defined in the road design specifications. Concretely, the filtered image $I_{1}^{\prime \prime}$, as illustrated in Figure 1, is obtained using the following equation:

$$
\begin{aligned}
I_{1}^{\prime \prime}(u, v)=2 I^{\prime}(u, v) & -\left|I^{\prime}(u, v-w)+I^{\prime}(u, v+w)\right| \\
- & \left|I^{\prime}(u, v-w)-I^{\prime}(u, v+w)\right|
\end{aligned}
$$

As discussed in [20]-[21], this filter is robust against shadows and road irregularities, such that asphalt resurfacing, potholes, etc. In addition, it is less prone to errors when compared to other lane marking detectors in the literature such as the ones proposed in [22], [23].

After image filtering, the new image $I_{1}^{\prime \prime}$ is thresholded and re-filtered to respectively keep only the lane object and remove the small blobs. In $I_{1}^{\prime \prime}$, the previous filter has amplified the gray intensity contrast between the road marker nearby white defined by $I_{1}^{\prime \prime}(u, v)=255$ and the rest of the image close to black $I_{1}^{\prime \prime}(u, v)=0$. Then, applying a threshold wisely chosen, we can compute a binary image $I_{2}^{\prime \prime}$ which aims to keeps only the road lane pixels as true and the rest as false as illustrated by the step 3 in Figure 1. Although, the first filter was supposed to retain only the road markers, some white blobs appear on the binary image $I_{2}^{\prime \prime}$ due to high gray intensity contrast between other objects than lanes in $I$. To cope with this issue, a second filtering level is applied in order to remove the white blobs. If we assume that they correspond to small isolated regions of few white pixels compared to the size of the road markers, it comes an intuitive and simple filter. It computes the number of connected white pixels and then erase the white blobs through an area thresholding. An example of resulting image is shown in image $I^{\prime \prime}$ on Figure 1.

Once the binary image of the road lane markers $I^{\prime \prime}$ is obtained, we aim separate the lanes (left, center and right in our case). To that end, a sliding window algorithm is used to track each lane independently in $I^{\prime \prime}$. Note that this method is illustrated by the step 5 on Figure 1 and allows to track both straight and curved lanes since the windows can horizontally slide. For each new frame, a first initialization step aims to detect the pixel position of each lane at the bottom of the image thanks to the histogram technique. It simply counts the number of white pixel for each column in the considered area and allows to place the first corresponding widows. Then, the same pattern is used to initialize the lateral position of each new window which is slid regarding the histogram result in the previous one. The window size is chosen such that its width does not capture two lanes at the same time and its height small enough to ensure curved lanes tracking.

At the same time, the image $I^{\prime \prime}$ is re-scaled and moved from the image frame coordinate $\mathcal{F}_{i}$ to the vehicle coordinate system $\mathcal{F}_{v}$ whose origin is the projection of the camera center on the ground and the $X$-axis corresponds to the vehicle longitudinal axis. Indeed, in the BEV images $\left(I^{\prime}-I^{\prime \prime}\right)$ all the object distances are expressed in pixel whereas, in our case, the desired unit is the meter. It means a pixel located at $(u, v)$ in $\mathcal{F}_{i}$ is moved to $(x, y)$ in $\mathcal{F}_{v}$ such that $x=X_{\max }-$ $v \frac{X_{\max }-X_{\min }}{n}$ et $y=Y_{\max }-u \frac{Y_{\max }-Y_{\min }}{m}$.

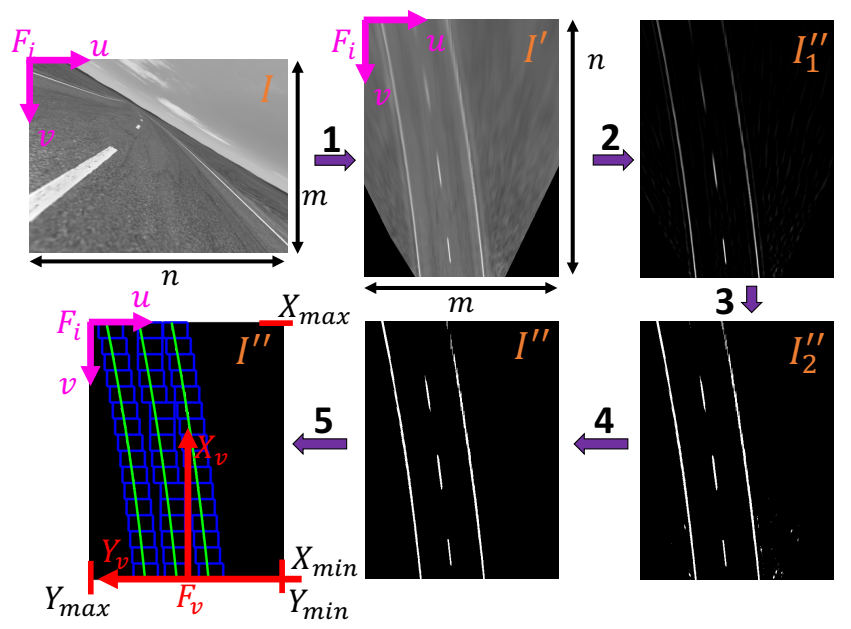

Fig. 1: Five steps to obtain the lane fitting from a captured frame

A this point, we have a scatter graph in the vehicle coordinate system $F_{v}$ for each detected lane which is going to be the input of the fitting step discussed in the next section.

\section{LATERAL POSITION ESTIMATION AND ROAD CURVATURE PREDICTION}

This section describes an approach to estimate the P2WV lateral position, its steering behavior and to predict the road curvature.

Civil engineers have largely adopted and optimized the clothoid model, also well-known under the name of Euler spiral, to design road [24]. The clothoid-based design ensures smooth transitions between straight and curved lines helping drivers to track the road lane markers and avoiding abrupt changes in steering direction. The clothoid is defined such that the length of the curve $l$ from the curve origin must be proportional to the curvature. Hence, the following expression was introduced in the road design literature [25]:

$$
C(l)=C_{0}+C_{1} l
$$

where $C_{0}$ and $C_{1}$ are respectively the initial curvature and its rate along the curve. Note that $C_{0}=0$ and $C_{1}=0$ 
correspond to a straight line, $C_{0} \neq 0$ and $C_{1}=0$ to a circular curve and $C_{0} \neq 0$ and $C_{1} \neq 0$ to a clothoid. This model is only valid for horizontal curves when the road is planar, in other words without any elevation. As in [25], if we consider the heading angle between the road tangent and the vehicle $X$-axis (see Figure 2) is small (less than $10^{\circ}$ ), the equation (7) can be approximated in the Cartesian coordinate system with the expression:

$$
y(x) \approx Y_{0}+\tan (\Delta \psi) x+\frac{1}{2} C_{0} x^{2}+\frac{1}{6} C_{1} x^{3}
$$

with $Y_{0}$ the lateral offset, $\Delta \psi$ the vehicle relative heading angle to the road trajectory, $C_{0}$ and $C_{1}$ the clothoid parameters introduced in (7). Figure 2 depicts the parameter layout. Most of the time, the road markers of extra-urban and highway roads are supposed to be parallel. In this context, if we consider the right, center and left lanes, their corresponding models (8) will only differ from their corresponding offsets $Y_{0}$ respectively denoted $Y_{0_{r}}, Y_{0_{c}}$ and $Y_{0_{l}}$.

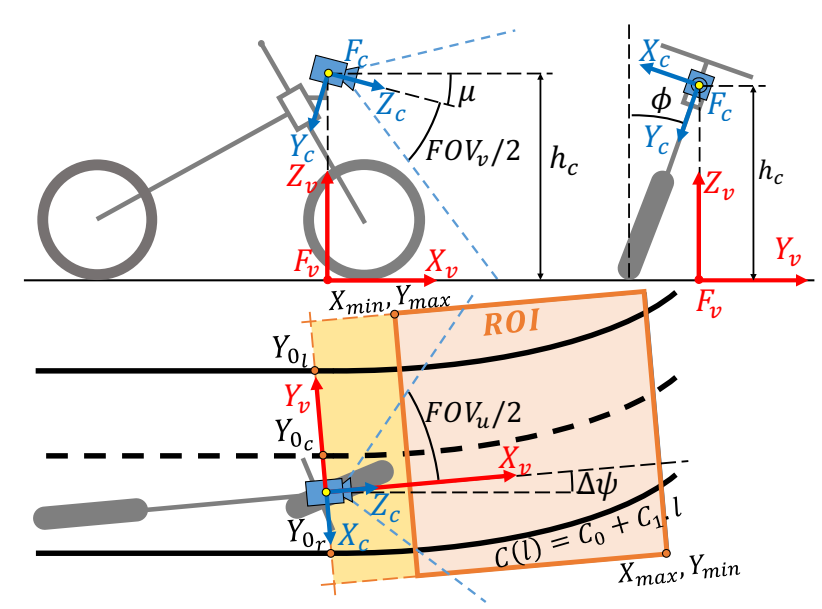

Fig. 2: IPM \& clothoid model

Then, once each lane is detected by the sliding window technique in image $I^{\prime \prime}$, it is approximated by a 3 rd order polynomial function in order to identify the clothoid parameters given in equation (8). An example of fitting result is shown in Figure 1 on image $I^{\prime \prime}$. As we can see, even if the approximated third order polynomial curves in green are not exact they still fit perfectly the road marker shapes. Note that because of the camera FOV limits and its small mounting tilt $\mu_{0}$, it introduces a blind spot area in the first few meters in front of the vehicle (see yellow region in Figure 2). This last can not be reconstructed in the BEV since it is not captured by the real camera. That is why we define the minimum ROI distance $X_{\min }>0$ such that the blind spot area is not considered in the BEV. Nevertheless, once the lanes are approximated with the third polynomial functions we can reconstruct the road lane shapes in the blind spot region since $X_{\min }$ is negligible compared to the minimum distance for significant road trajectory change. In the end, we can estimate the dynamics at $X=0 \mathrm{~m}$ which corresponds to the camera center projection on the ground.
This section ends the algorithm synthesis which creates a BEV of a captured frame, filters the road markers and track them to separate the different lanes. Then, third order fitting functions allow the identification of the clothoid model parameters for each tracked lane. The identified parameter $Y_{0}$ directly give us the relative motorcycle lateral position with respect to the considered lane, $\tan (\Delta \psi)$ expresses the P2WV steering behavior (neutral, over or under-steering), $C_{0}$ and $C_{1}$ give information about the horizontal road geometry forward the vehicle.

\section{SIMULATION RESULTS}

This section validates the proposed algorithm on various scenarios using the advanced motorcycle simulator BikeSim.

The progress in vehicle simulator sofware allows to simultaneously simulate the complete non-linear vehicle dynamics and create virtual cameras to test computer vision algorithms. The recent literature [26], [27] shown it becomes common to use these advanced simulators like CarSim, CarMaker, etc. to perform a first validation step. Indeed, these tools attract more and more interest in the context of ADAS and ARAS development since they allow to test dangerous driving scenario without any real risk. In this work, the simulations have been carried out with BikeSim which is the motorcycle version of CarSim.

The camera has been virtually installed in front of the P2WV at a height $z_{c}=1.10 \mathrm{~m}$ and mechanically titled of an angle $\mu_{0}=15^{\circ}$. Its recording speed is set at $30 \mathrm{fps}$ which is a very basic frame rate for conventional camera. In the simulations below, the results are compared between two different standard image sizes, a low resolution $(640 \times 480)$ and a higher of $(1080 \times 720)$. For both resolutions the camera vertical and horizontal FOV are respectively equal to $F O V_{v}=58.4^{\circ}$ and $F O V_{u}=80^{\circ}$. The ROI is chosen large enough to capture the left, center and right lanes with $Y_{\max }=-Y_{\min }=15 \mathrm{~m}$. As discussed previously $X_{\max }=30 \mathrm{~m}$ and $X_{\min }=5 \mathrm{~m}$ to respectively let the time to the rider to react in case of danger and to avoid blind spot consideration. Note that all the results below are given at $X_{0}$ which is the projection of the camera center on the ground.

The proposed algorithm was tested under different scenarios and two of them are presented. In these two scenarios, we assume the road to be planar (no elevation) and at least the right lane is structured and detectable. For the simulations below, this lane is considered as the reference since in most cases it is closer to the $\mathrm{P} 2 \mathrm{WV}$ for right-hand drive and it is a solid lane. Nevertheless, it is utterly possible to consider the center line or the left one even if it is a dashed lane but the estimation performances could be degraded.

\section{A. Scenic road}

A constant speed scenic road simulation at $100 \mathrm{~km} / \mathrm{h}$ is presented first. This scenario simulates an extra-urban road which is composed of straight lines, several circular turns and clothoids to ensure smooth connections (see Figure 3). This kind of scenario is particularly interesting in the development of ARAS since it is one of the most deadly. This is because 
of the ratio between road curvature and speed limits. Indeed, urban roads can have more aggressive turns but the speed limit is slower although on a highway the speed limit is faster but the curve radius are very large. In addition, with its large roll angles as illustrated in Figure 3, this simulation validates one of the paper's contributions, namely the development of an IPM formulation for leaning vehicles.

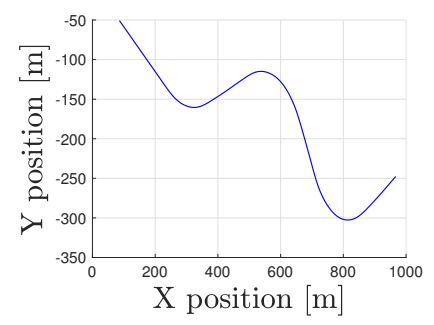

(a) Vehicle trajectory

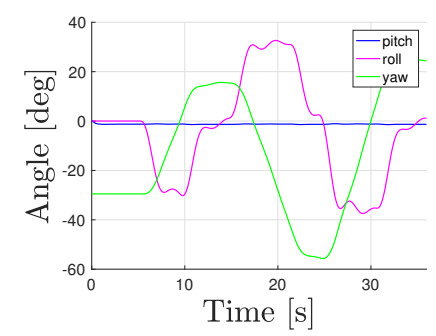

(b) Vehicle Euler angles
Fig. 3: Scenic road scenario at $100 \mathrm{~km} / \mathrm{h}$

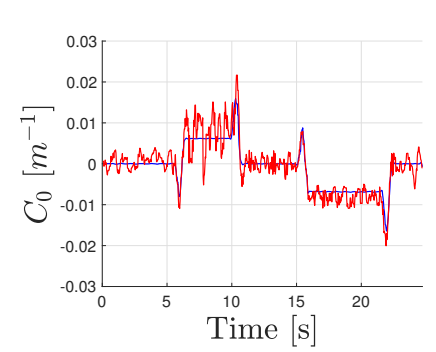

(a) Clothoid parameter $C_{0}$

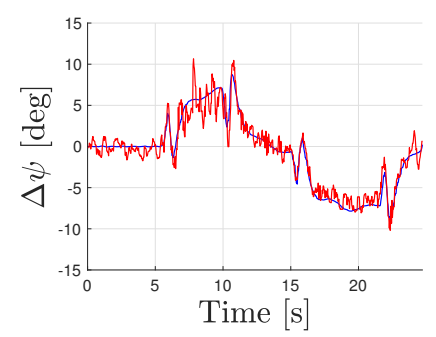

(c) Relative heading angle

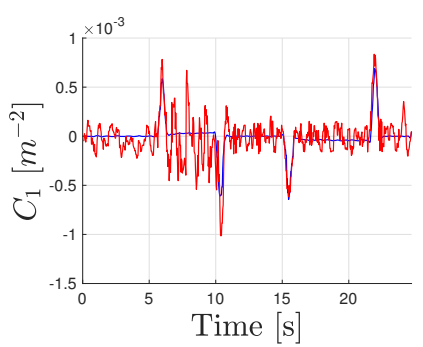

(b) Clothoid parameter $C_{1}$

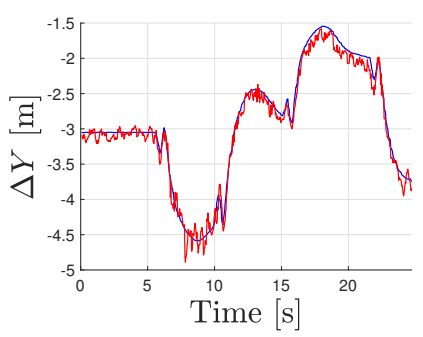

(d) Relative lateral position
Fig. 4: Scenic road parameter estimations with a (640x480) camera resolution

Figure 3 depicts the vehicle trajectory along the scenic road scenario and the corresponding motorcycle roll, yaw and pitch angles. Whereas Figures 4 and 5 show the estimated parameters, in red, and the actual ones in of the clothoid model given in (8) with regard to the right lane. We can see for both resolutions the estimations are affected by noise coming from the image processing uncertainties and the polynomial approximation which are related to the marker width generally around $20 \mathrm{~cm}$. Nevertheless, as we can remark between Figure 4 and 5 where the camera resolutions are respectively $(640 \times 480)$ and $(1080 \times 720)$, increasing the resolution significantly contribute to reduce the noise. But we must keep in mind, that an increase in the image resolution has serious consequences on the computation time.

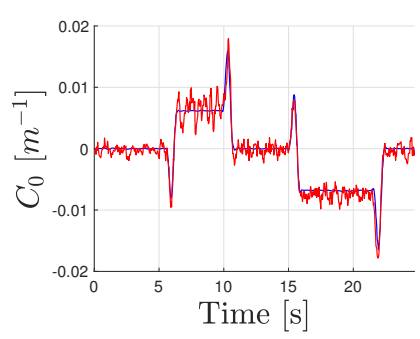

(a) Clothoid parameter $C_{0}$

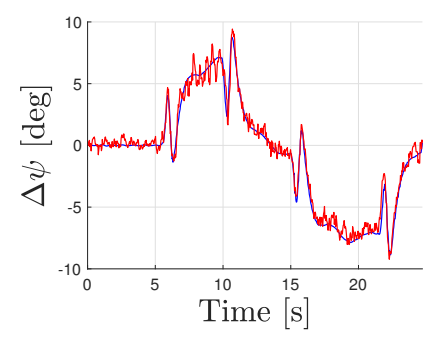

(c) Relative heading angle

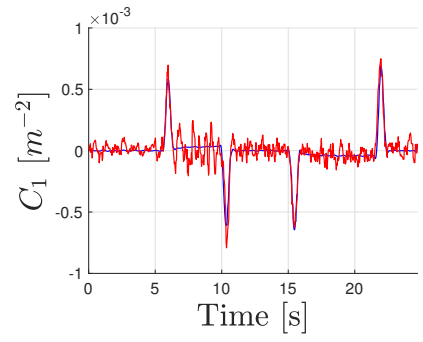

(b) Clothoid parameter $C_{1}$

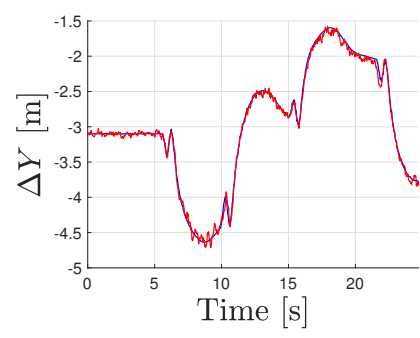

(d) Relative lateral position
Fig. 5: Scenic road parameter estimations with a (1080x720) camera resolution

\section{B. Double lane change}

The Double Lane Change (DLC) is a well-known scenario in the development of vehicle safety systems since it is an emergency maneuver for obstacle avoidance.

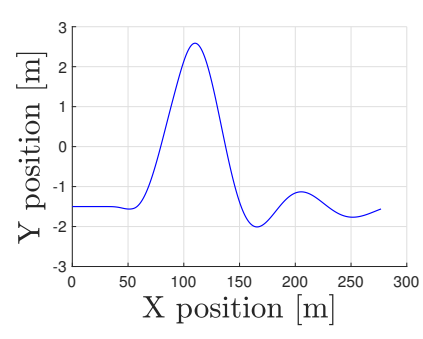

(a) Vehicle trajectory

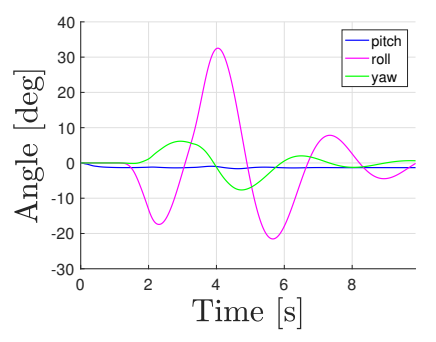

(b) Vehicle orientation
Fig. 6: Double lane change scenario at $100 \mathrm{~km} / \mathrm{h}$

It is also used to simulate an overtaking scenario. In addition to test the proposed algorithm on an emergency riding scenario, this scenario allows to test a very specific case too. Indeed, the road is straight whereas the vehicle has a large lateral deviation which is a rare case where the vehicle trajectory significantly differs from the road route. Like for the scenic road the riding speed is assumed constant at $100 \mathrm{~km} / \mathrm{h}$. Figure 6. a depicts the P2WV trajectory during the DLC, the vehicle is initially in a straight trajectory and suddenly crosses the center lane to avoid an obstacle before going back to its initial lateral position. Figure 6.b shows that even if the road shape is straight, a DLC maneuver with a P2WV highly excites the lateral dynamics.

As for the scenic road, Figures 7 and 8 present the estimation results respectively for a $(640 \times 480)$ and $(1080 \times 720)$ camera resolution. The estimations are plotted in red whereas the actual clothoid model parameters are in blue. 


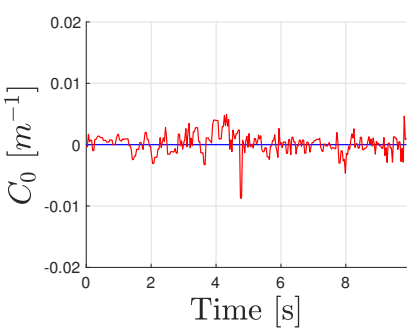

(a) Clothoid parameter $C_{0}$

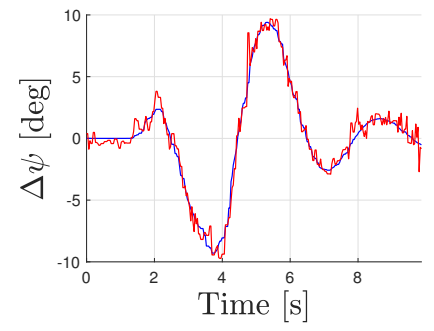

(c) Relative heading angle

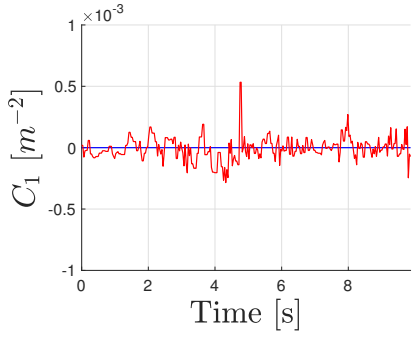

(b) Clothoid parameter $C_{1}$

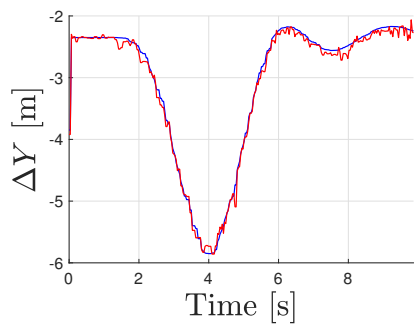

(d) Relative lateral position
Fig. 7: DLC parameter estimations with a $(640 \times 480)$ camera resolution

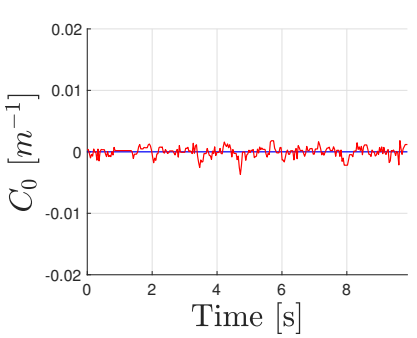

(a) Clothoid parameter $C_{0}$

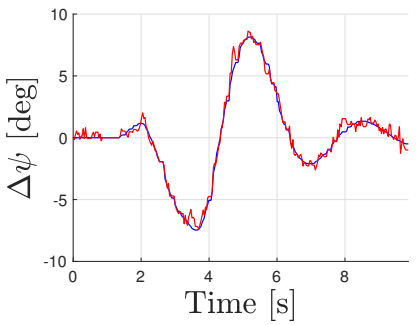

(c) Relative heading angle

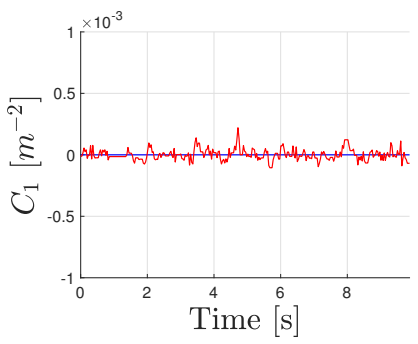

(b) Clothoid parameter $C_{1}$

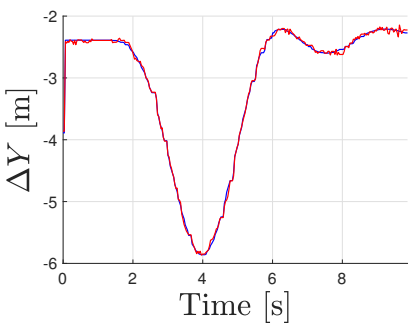

(d) Relative lateral position
Fig. 8: DLC parameter estimations with a (1080x720) camera resolution

As expected, the clothoid parameters $C_{0}$ and $C_{1}$ are zero because of the straight road markers. Moreover, in this specific case, the P2WV lateral position as depicted in Figure 7.d and 8.d corresponds directly to the DLC trajectory with a constant offset and the relative heading angle shown in 8.c and 7.c to the absolute vehicle yaw. As for the previous results, the estimations are affected by noise whose amplitude decreases when increasing camera resolution. We can remark, for a same resolution, the estimation noise amplitude is smaller for the DLC than for scenic road scenario. It confirms that the noise is mainly due to polynomial approximations and lane detection uncertainties which are higher in curves.
Moreover, it verifies that even if we keep a fixed reference, the right lane in our case, while the vehicle is on the left road side the relative lateral position estimation is still accurate.

\section{Result discussion}

This subsection presents a Root-Mean-Square Error (RMSE) study which is a well-known technique to contrast the estimation errors. In Table I, the RMSE is computed for both camera resolutions $((640 \times 480)$ and $(1080 \times 720))$ and both scenarios. Let $y(t)$ be a signal and $\hat{y}(t)$ its estimation, the RMSE expression is given by: $R M S E=$ $\sqrt{\frac{1}{n} \sum_{i=1}^{n}\left(y_{i}(t)-\hat{y}_{i}(t)\right)^{2}}$. It is proportional to the square of the estimation error, consequently the lower the RMSE is the better is the estimation performance.

\begin{tabular}{|c|c|c|c|}
\hline & & \multicolumn{2}{|c|}{ RMSE } \\
\hline & Resolution & $(640 x 480)$ & $(1080 \times 720)$ \\
\hline \multirow{5}{*}{ Scenic Road } & $C_{0}\left[m^{-1}\right]$ & $2.23 e^{-3}$ & $1.04 e^{-3}$ \\
\hline & $C_{1}\left[m^{-2}\right]$ & $12.6 e^{-5}$ & $5.80 e^{-5}$ \\
\hline & $\Delta \psi[\operatorname{deg}]$ & $9.58 e^{-1}$ & $4.94 e^{-1}$ \\
\hline & $Y_{0}[m]$ & $8.02 e^{-2}$ & $4.30 e^{-2}$ \\
\hline & $\tau[\%]$ & 98.0 & 202.2 \\
\hline \multirow{5}{*}{ DLC } & $C_{0}\left[m^{-1}\right]$ & $1.90 e^{-3}$ & $0.81 e^{-3}$ \\
\hline & $C_{1}\left[m^{-2}\right]$ & $1.08 e^{-4}$ & $0.46 e^{-4}$ \\
\hline & $\Delta \psi[\operatorname{deg}]$ & $8.12 e^{-1}$ & $4.10 e^{-1}$ \\
\hline & $Y_{0}[m]$ & $7.28 e^{-2}$ & $3.40 e^{-2}$ \\
\hline & $\tau[\%]$ & 99.8 & 195 \\
\hline
\end{tabular}

TABLE I: RMSE RESULTS

In Table I, the parameter $\tau$ denotes the computation time performance as the ratio between the total estimation time and the simulation time at a recording speed of $30 \mathrm{fps}$. The simulation was carried out on macOS with a $3.1 \mathrm{GHz}$ Intel Core $17 \mathrm{CPU}$. Of course deploying the algorithm on a real-time OS with optimized image processing toolboxes could give much better performances but this first study gives a first idea about the algorithm speed. The results shows the potential of the proposed algorithm since it is as of now real-time for a $(640 \times 480)$ resolution even if the OS is not optimized. Nevertheless, increase the camera resolution undoubtedly increase the estimation accuracy by decreasing the noise but it has serious consequences on the computation time. Roughly speaking, the estimation error is divided by 2 when the resolution increase from $(640 \times 480)$ to (1080x720) whereas the computation time is multiplied by 2. Moreover, Table I endorses the fact that even for a low camera resolution as $(640 \times 480)$, the estimation accuracy is great. While commercial GPS have an accuracy about one meter and are not capable of measuring the relative heading angle with an acceptable precision, our solution provides P2WV lateral position and relative heading angle estimation, in real time, with an accuracy respectively about $10 \mathrm{~cm}$ and 2-3 degrees. 


\section{CONCLUSION}

This paper introduced a vision-based algorithm to simultaneously estimate the relative $\mathrm{P} 2 \mathrm{WV}$ lateral position, its relative steering behavior (relative yaw angle) to the road markers, and predict the road curvature forward the motorcycle. The proposed solution is based on the well-known IPM technique which was adapted for leaning vehicles and a filtering step to keep only the road markers in the BEV image. Sliding windows are then utilized to track independently each road lanes and finally, third order fitting functions were used to identify the clothoid model parameters. These last are the road curvature radius $C_{0}$, its rate $C_{1}$, the relative yaw $\Delta \psi$ and the relative lateral position $Y_{0}$ to the road markers. The solution has been tested on various test scenarios including a scenic road and a DLC with two camera resolution of (640x480) and (1080x720). Furthermore, a RMSE study shows a great performance of the algorithm. Even for a low camera resolution of $(640 \times 480)$, the results are much better than the measures obtained with a common GPS.

Finally, in the context of ARAS development, the main contributions of this paper is the development of a realtime vision-based algorithm using only a basic camera and an IMU to estimate and predict crucial information when turning. It is able to estimate the P2WV lateral position on the road and its steering behavior allowing to detect over or under-steering. Last but not least, the algorithm allows to predict the road curvature within $30 \mathrm{~m}$ ahead of the vehicle which provides sufficient time to the rider to react in case of a problem (speeding in curve, etc.). In addition, a specific formulation of the IPM were introduced in equation (5) for leaning vehicles.

The discussed results are very promising and open lot of possibilities about the use of vision in ARAS developments. The next step will deal with the embedded architecture design and the algorithm optimization to perform real time validation on experimental data. In the near future, we would like to address other steps of ARAS development such that the risk function and the rider alert system designs. In addition, the algorithm could be paired with our previous work on observers to bypass the use of the IMU.

\section{REFERENCES}

[1] NHTSA, "2016 fatal motor vehicle crashes: Overview," U.S. Department of Transportation National Highway Traffic Safety Administration, Tech. Rep., 2017.

[2] I. William Schneider, P. Savolainen, and D. Moore, "Effects of horizontal curvature on single-vehicle motorcycle crashes along rural two-lane highways," Transportation Research Record: Journal of the Transportation Research Board, vol. 2194, pp. 91-98, 2010.

[3] D. D. Clarke, P. Ward, C. Bartle, and W. Truman, "In-depth study of motorcycle accidents," School of Psychology, University of Nottingham, Tech. Rep., November 2004.

[4] R. Risack, N. Mohler, and W. Enkelmann, "A video-based lane keeping assistant," IEEE Intelligent Vehicles Symposium, pp. 356-361, 2000.

[5] J. Michel, A. Saxena, and A. Y. Ng, "High speed obstacle avoidance using monocular vision and reinforcement learning," International Conference on Machine Learning, pp. 593-600, 2005.

[6] M. Barnada, C. Conrad, H. Bradler, M. Ochs, and R. Mester, "Estimation of automotive pitch, yaw and roll using enhanced phase correlation on multiple far-field windows," Intelligent Vehicles Symposium, June 2015.
[7] C.-Y. Fang, W.-H. Hsu, C.-W. Ma, and S.-W. Chen, "A vision-based safety driver assistance system for motorcycles on a smartphone," Conference on Intelligent Transportation Systems, pp. 328-333, Oct 2014.

[8] L. Gasbarro, A. Beghi, R. Frezza, F. Nori, and C. Spagnol, "Motorcycle trajectory reconstruction by integration of vision and mems accelerometers," Conference on Decision and Control, pp. 779-783, Dec 2004.

[9] A.Guarnieri, F. Pirotti, and A. Vettore, "Low-cost mems sensors and vision system for motion and position estimation of a scooter," Sensors, vol. 13, pp. 1510-22, 022013.

[10] F. Nori and R. Frezza, "Accurate reconstruction of the path followed by a motorcycle from the on-board camera images," IEEE Intelligent Vehicles Symposium, pp. 259-264, June 2003.

[11] M. Schlipsing, J. Schepanek, and J. Salmen, "Video-based roll angle estimation for two-wheeled vehicles," IEEE Intelligent Vehicles Symposium, pp. 876-881, June 2011.

[12] M. Schlipsing, J. Salmen, B. Lattke, K. G. Schröter, and H. Winner, "Roll angle estimation for motorcycles: Comparing video and inertial sensor approaches," IEEE Intelligent Vehicles Symposium, pp. 500505, June 2012.

[13] F. Biral, M. D. Lio, R. Lot, and R. Sartori, "An intelligent curve warning system for powered two wheel vehicles," European Transport Research Review, vol. 2, no. 3, pp. 147-156, December 2010.

[14] P. Damon, H. Dabladji, D. Ichalal, L. Nehaoua, and H. Arioui, "Estimation of lateral motorcycle dynamics and rider action with luenberger observer," IEEE Intelligent Transportation Systems (ITSC), pp. 2392-2397, 2016.

[15] P. M. Damon, H. Dabladji, D. Ichalal, L. Nehaoua, H. Arioui, and S. Mammar, "Lateral motorcycle dynamics and rider action estimation: An lpv unknown input observer approach," in 2016 IEEE Conference on Control Applications (CCA), Sept 2016, pp. 711-716.

[16] P. M. Damon, D. Ichalal, H. Arioui, and S. Mammar, "Cascaded flatness-based observation approach for lateral motorcycle dynamics estimation," Conference on Systems, Man, and Cybernetics (SMC), pp. 3243-3248, Oct 2017.

[17] P.-M. Damon, D. Ichalal, L. Nehaoua, and H. Arioui, "Lateral \& steering dynamics estimation for single track vehicle: Experimental tests," World Congress on International Federation of Automatic Control (IFAC), vol. 50, no. 1, pp. 3400 - 3405, 2017.

[18] M. E.-H. Dabladji, D. Ichalal, H. Arioui, S. Mammar, and L. Fridman, "Estimation of lateral dynamics and road curvature for two-wheeled vehicles: A hosm observer approach," World Congress on International Federation of Automatic Control (IFAC), vol. 47, no. 3, pp. 2806-2811, August 2014.

[19] E. Malis and M. Vargas, "Deeper understanding of the homography decomposition for vision-based control," INRIA, Tech. Rep., 2007.

[20] M. Nieto, J. Arróspide Laborda, and L. Salgado, "Road environment modeling using robust perspective analysis and recursive bayesian segmentation," Machine Vision and Applications, vol. 22, no. 6, pp. 927-945, November 2011.

[21] Y. W. Seo and R. R. Rajkumar, "Utilizing instantaneous driving direction for enhancing lane-marking detection," IEEE Intelligent Vehicles Symposium, pp. 170-175, June 2014.

[22] M. Bertozzi and A. Broggi, "Gold: a parallel real-time stereo vision system for generic obstacle and lane detection," IEEE Transactions on Image Processing, vol. 7, no. 1, pp. 62-81, January 1998.

[23] M. Nieto, L. Salgado, F. Jaureguizar, and J. Cabrera, "Stabilization of inverse perspective mapping images based on robust vanishing point estimation," Intelligent Vehicles Symposium, pp. 315-320, June 2007.

[24] G. Casal, D. Santamarina, and M. E. Vazquez-Mendez, "Optimization of horizontal alignment geometry in road design and reconstruction," Transportation Research Part C: Emerging Technologies, vol. 74, pp. 261-274, January 2017.

[25] E. D. Dickmanns and B. D. Mysliwetz, "Recursive 3-d road and relative ego-state recognition," IEEE Transactions on Pattern Analysis and Machine Intelligence, vol. 14, no. 2, pp. 199-213, February 1992.

[26] G. Cario, A. Casavola, G. Franze, and M. Lupia, "Data fusion algorithms for lane departure warning systems," American Control Conference, pp. 5344-5349, June 2010.

[27] C. M. Kang, S. H. Lee, and C. C. Chung, "Lane estimation using a vehicle kinematic lateral motion model under clothoidal road constraints," IEEE Conference on Intelligent Transportation Systems, pp. 1066-1071, Oct 2014. 